\title{
Efecto de la metionina en una dieta integral de yuca para ovinos en la desintoxicación de ácido cianhídrico
}

\author{
Comunicación Breve
}

Fernando Duarte ${ }^{1}$, Carlos Sandoval-Castro².

${ }^{1}$ Instituto Nacional de Investigaciones Forestales Agrícolas y Pecuarias - Campo Tizimín, Tizimín, Yucatán. ${ }^{2}$ Facultad de Medicina Veterinaria y Zootecnia, Universidad Autónoma de Yucatán, Mérida, Yucatán, México.

\section{RESUMEN.}

Introducción. El proceso de desintoxicación de los glúcidos cianogénicos contenidos en la yuca (Manihot esculenta) involucra procesos metabólicos que requieren azufre. La yuca es deficiente en aminoácidos azufrados y estos podrían estar limitando la capacidad de los ovinos para metabolizar este tipo de compuestos.

Objetivo. Evaluar el empleo de metionina suplementaria como mecanismo para facilitar la desintoxicación de ácido cianhídrico en ovinos consumiendo dieta de yuca integral ensilada.

Materiales y métodos. Se emplearon 3 ovinos (40.7 $\mathrm{kg}$ peso vivo promedio) en un diseño de cambio, con 2 periodos de 20 d (10 d adaptación, $5 \mathrm{~d}$ dieta testigo, $5 \mathrm{~d}$ dieta experimental). Los ovinos fueron mantenidos en jaulas metabólicas y alimentados con yuca ensilada ad líbitum. Se emplearon 2 dietas: a) dieta testigo a base de yuca ensilada y b) dieta experimental - ensilaje $+20 \mathrm{~g}$ de metionina/d. Se midió el consumo voluntario de ensilaje y se colecto la orina para medir la excreción diaria de tiocianato. Los resultados fueron analizados empleando el proceso linear generalizado (GLM) considerando animal y periodo como fuentes de variación para la partición del error.

Resultados. Ambas dietas tuvieron consumos semejantes ( $\mathrm{p}>0.05$ ) de yuca ensilada (c. 930gMS/ d). La excreción diaria de tiocianato fue de 11.6 vs $28.8 \pm 5.42 \mathrm{mg} / \mathrm{d}$, con y sin metionina respectivamente $(\mathrm{p}=0.067)$.

Discusión. La metionina probablemente juega un papel de donador de azufre necesario en el metabolismo de desintoxicación de los GCN. La adición de metionina a una dieta de yuca ensilada puede ayudar al proceso de desintoxicación de los GCN. Estudios adicionales deben confirmar estos hallazgos. (Rev Biomed 2002; 13:116-119)

Palabras clave: Yuca integral ensilada, Manihot esculenta, desintoxicación, ovinos, metionina, ácido cianhídrico.

Solicitud de sobretiros: Dr. Carlos A. Sandoval-Castro, Departamento de Nutrición Animal, Facultad de Medicina Veterinaria y Zootecnia, Universidad Autónoma de Yucatán, Apdo. Postal 4-116 Itzimná, C.P. 97100, Mérida, Yucatán, México. 


\section{F Duarte, C Sandoval-Castro.}

\section{SUMMARY.}

Efect of methionine supplements in sheep fed on whole cassava silage diet to improve cyanide detoxification.

Introduction. The detoxification of the cyanogenic glucosides (HCN) contained in cassava (Manihot esculenta) involves metabolic pathways which require sulphur. Cassava is deficient in sulphur and sulphur-amino acids and hence they maybe limiting its metabolism in sheep.

Objective. To evaluate the use of supplementary methionine as a mechanism to facilitate $\mathrm{HCN}$ detoxification in sheep fed whole cassava silage.

Materials and methods. Three sheep $(40.7 \mathrm{~kg}$ average live weight) were employed in a changeover design in 2 periods of $20 \mathrm{~d}$ (10 d adaptation, $5 \mathrm{~d}$ control diet, $5 \mathrm{~d}$ experimental diet). Diets were a) whole cassava silage only, b) whole cassava silage $+20 \mathrm{~g}$ methionine/d. Animals were kept in metabolic crates and fed whole cassava silage ad libitum. Voluntary intake and urinary excretion of thiocyanate were measured daily. Data was analysed with the general lineal model (GLM) taking into account animal and period for model error partition.

Results. Both diets had similar voluntary intakes ( $\mathrm{p}>0.05)$ of cassava silage (c. $930 \mathrm{gMS} / \mathrm{d})$. Methionine caused an increase $(\mathrm{p}=0.067)$ in the urinary excretion of thiocyanate (11.6 vs $28.8 \pm$ $5.42 \mathrm{mg} / \mathrm{d}$, with and without methionine respectively).

Discussion. Methionine is probably playing the role of sulphur donor in HCN detoxification process. Methionine supplements in whole cassava silage diets might help to improve its utilization by helping sheep detoxify HCN. Additional studies are needed to confirm these findings.

(Rev Biomed 2002; 13:116-119)

Key words: Whole cassava silage, detoxification, sheep, Manihot esculenta, methionine, cyanogenic glucosides.

\section{INTRODUCCIÓN.}

El bajo valor nutricio de los pastos tropicales empleados en la alimentación de rumiantes hace necesaria la búsqueda de suplementos de bajo costo y mayor valor nutricio. La yuca presenta un gran potencial para su uso como complemento, sólo limitada por su contenido de compuestos tóxicos como los glúcidos cianogénicos (linamarina y lotaustralina) (1), y su disponibilidad estacional la cual puede ser solventada empleando el proceso de ensilaje (2).

La yuca como alimento posee el potencial de inducir intoxicación por ácido cianhídrico (HCN), el cual es liberado al hidrolizarse los glúcidos cianogénicos contenidos en ésta. Se considera que el principal mecanismo de desintoxicación del HCN es la conversión de dicho producto en tiocianato, el cual es excretado en orina. Esta conversión se realiza especialmente en el hígado en presencia de azufre coloidal. Existen varias moléculas en el organismo que pueden proveer azufre para el proceso (metionina, cistina, cisteina) (3). La yuca ha sido reportada consistentemente como un alimento de bajo contenido en amino ácidos azufrado (4-10). Esto hace evidente la necesidad de metionina adicional en dietas basadas en yuca dado su papel potencial como fuente de azufre en la desintoxicación de HCN.

En trabajos recientes, bovinos alimentados con yuca ensilada y suplementados con metionina han mostrado una mejor respuesta productiva (11). Este comportamiento observado llevó a la realización del presente trabajo para explicar el modo de acción de la metionina en dietas de yuca ensilada, empleando el ovino como un modelo de rumiante. Adicionalmente en una búsqueda retrospectiva de 5 años no se encontraron reportes sobre el uso de metionina en rumiantes alimentados con dietas a base de ensilaje de yuca. Por lo que el objetivo del presente trabajo fue estudiar el empleo de metionina como mecanismo para facilitar la desintoxicación de HCN en dietas de yuca ensilada.

\section{Revista Biomédica}


Metionina para ovinos en dietas de yuca ensilada.

\section{MATERIALES Y MÉTODOS.}

Se emplearon 3 ovinos machos de $40.7 \pm 1.5$ $\mathrm{kg}$ peso vivo en un diseño de cambio (change over) con dos periodos de 20 días, 10 días de adaptación, 5 días de mediciones en dieta testigo y 5 días de mediciones en dieta experimental. Los borregos fueron mantenidos en cajas metabólicas individuales, las cuales permitieron la medición del consumo voluntario y la recolección total de orina.

Los animales fueron alimentados todo el periodo experimental con ensilado de yuca integral ad líbitum empleando el siguiente esquema.

A.- Días 1 a 10, periodo de adaptación a la yuca ensilada.

B.- Días 11 a 15, dieta testigo. Medición de consumo voluntario mediante el pesado del alimento ofrecido y rechazado, una muestra fue tomada para análisis de materia seca (MS). Diariamente, se colectó la orina total y se tomo una alícuota de $100 \mathrm{~mL}$ que fue conservada en congelación hasta su análisis en el laboratorio.

C.- Días 16 al 20, dieta experimental suplementada con $20 \mathrm{~g}$ de metionina; se realizaron las mismas mediciones que en el periodo anterior.

Al término de los 20 días los animales fueron alimentados con heno de pasto estrella (Cynodon nlemfluensis) por 15 días. Transcurridos estos, se reinició el procedimiento experimental descrito previamente por un nuevo periodo de 20 días. Análisis de laboratorio.

Las muestra de yuca ensilada fue secada en estufa de ventilación forzada a $60^{\circ} \mathrm{C}$ para conocer su contenido de MS. Las muestras de orina fueron analizadas para tiocianato de acuerdo a la metodología descrita en la norma Codex CAC/ GL13 (12).

Análisis estadístico.

Los resultados fueron analizados en un diseño de cambio, empleando el procedimiento linear generalizado (GLM) del paquete estadístico Minitab 12 (13), considerando el efecto de animal y periodo como fuentes de variación. Se contó con 6 replicas por tratamiento y 7 grados de libertad para el error.

\section{RESULTADOS.}

Los animales consumieron cantidades semejantes $(\mathrm{p}=0.4)$ de ensilaje de yuca $(\mathrm{kgMS} / \mathrm{d})$ con y sin el suplemento de metionina. Sin embargo, existió una tendencia hacia una mayor excreción $(\mathrm{p}=0.06)$ de tiocianato $(\mathrm{mg} / \mathrm{d}$ ía) cuando la metionina fue adicionada a la dieta (cuadro 1).

\section{Cuadro 1}

Consumo de ensilaje de yuca integral y excreción de tiocianato en orina de ovinos con y sin suplemento de metionina.

\begin{tabular}{lccc}
\hline Tratamiento & $\mathrm{N}$ & $\begin{array}{l}\text { Consumo } \\
(\mathrm{g} \text { MS/día })\end{array}$ & $\begin{array}{l}\text { Tiocianato } \\
(\mathrm{mg} / \mathrm{d})\end{array}$ \\
\hline Sin metionina & 6 & 916 & 11.6 \\
Con metionina & 6 & 966 & 28.8 \\
$\quad(20 \mathrm{~g} /$ día $)$ & & & \\
EEM & & 40.5 & 5.42 \\
$\mathrm{P}$ & & 0.411 & 0.067 \\
\hline
\end{tabular}

MS:materia seca, EEM: error estándar de la media

\section{DISCUSIÓN.}

A pesar de su alto contenido de $\mathrm{HCN}$ existen pocos casos documentados de intoxicación por yuca debido, tal vez, a que el mismo agente previene de alguna manera el consumo excesivo por los animales que tienen acceso a follaje de yuca (14). En el presente trabajo la dieta fue exclusivamente basada en yuca ensilada lo que evitó cualquier selección posible de la dieta. El proceso de ensilaje ayuda a reducir el contenido de glúcidos cianogénicos contenidos en la dieta al nivel adecuado para obtener consumos aceptables (3), permitiendo mantener a los animales con dietas basadas únicamente es este producto sin síntomas aparentes de intoxicación.

La eliminación tiocianato en la orina demostró que los animales llevan a cabo el proceso de desintoxicación de la dieta, pero que este fue favorecido por la adición de metionina suplementaria. La importancia de la adición de fuentes de azufre radica en la posibilidad de lograr 


\section{F Duarte, C Sandoval-Castro.}

la eliminación total de los compuestos resultantes del proceso de desintoxicación. La conversión de HCN a tiocianato representa una reducción 200:1 en la toxicidad del compuesto resultante (1), sin embargo el tiocianato es aún un potente bociogénico $(14,15)$ por lo que es importante su completa eliminación. No fue posible estimar en el presente ensayo si la cantidad de metionina adicionada fue suficiente para lograr este último propósito.

En conclusión, el empleo de metionina suplementaria en la ración de borregos puede ayudar al proceso de desintoxicación de los glúcidos cianogénicos contenidos en el ensilado de yuca integral. Por lo que se propone su inclusión en las raciones basadas en yuca. Sin embargo son necesarios estudios adicionales para confirmar este resultado, así como las cantidades necesarias para la completa eliminación de los metabólitos secundarios.

\section{AGRADECIMIENTOS.}

El presente trabajo fue financiado por CONACyTSISIERRA "Suplementación de toreste en pastoreo con yuca integral Manihot esculenta" convenio 980191.

\section{REFERENCIAS.}

1.- Ravindran V. Cassava leaves as animal feed: Potential and limitations. J Sci Food Agric 1993; 61:141-50.

2.- Moore CP, Cock JH. Cassava foliage silage as a feed source for Zebu calves in tropics. Trop Agric (Trinidad) $1985 ; 62: 142-4$.

3.- Buitrago JA. La yuca en la alimentación animal. Cali, Colombia: Centro Internacional de Agricultura Tropical; 1990.

4.- Rogers DJ, Milner M. Amino acid profile of manioc leaf protein in relation to nutritive value. Econ Bot 1963; 17:211-6.

5.- Eggum OL. The protein quality of cassava leaves. $\mathrm{Br} \mathrm{J}$ Nutr 1970; 24:761-9.

6.- Yeoh HH, Chew MY. Protein content and amino acid composition of cassava leaf. Phytochem 1976; 15:1597-9.
7.- Hutagalung RI. Additives other than methionine in cassava diets. En: Nestel B, Graham M, editores. Cassava as animal feed: Proceedings of a workshop, University of Guelph, Canada, International Development Research Centre. Ottawa, Canada; 1977. p. 18-32.

8.- Gomez G, Noma AT. The amino acid composition of cassava leaves, foliage, root tissue and whole-root chips. Nutr. Rep Int 1986; 33:595-601.

9.- Nwokolo E. Leaf meals from cassava (Manihot esculenta Crantz) and Siam weed (Eupatorium odoratum L.) As nutrient sources in poultry diets. Nutr. Rep Int 1977; 36:819-26.

10.- Ravindran G, Ravindran V. Changes in the nutritional composition of cassava (Manihot esculenta Crantz) leaves during maturity. Food Chem 1988; 27:299-309.

11.- Duarte VF, Pelcastre OA 2000. Adición de pasta de soya y metionina sobre el comportamiento de borregos alimentados con ensillaje de yuca. Memorias de la XXXVI Reunión Nacional de Investigación Pecuaria. 7-10 Noviembre, 2000, Hermosillo, Son., México. p. 197.

12.- CAC/GL 13. Codex Alimentarius Commission Guide Line 13. Programa conjunto FAO/OMS sobre normas alimentarias. Vol. 12. Normas codex sobre la leche y los productos lacteos. Roma: FAO 1991.

13.- Reference Manual, Release 12. Philadelphia: Minitab, State College; 1997.

14.- Swain T. Secondary compounds as protective agents. Ann Rev Plant Physiol 1977; 28:479-501.

15.- Langer P. Antithyriod action in rats of small doses of some naturally occurring compounds. Endocrinology 1966; 79:1117-22.

16.- Sihombing DTH, Cromwell GL, Hays VW. Effect of added thiocyanide and iodine to corn-soybean meal diets on performance and thyroid status of pigs. J Anim Sci 1971; 33:1154-9.

\section{Revista Biomédica}

